\title{
Improvement of Flexible Linear Stepping Actuator Driven by Pneumatic Balloons and Brakes
}

\author{
Yuya Eguchi, Tetsuya Akagi ${ }^{a}$, Shujiro Dohta and Wataru Kobayashi \\ Department of Intelligent Mechanical Engineering, Okayama University of Science, Ridai-cho, Kita-ku, Okayama, \\ 700-0005, Japan
}

\begin{abstract}
The development of soft actuator for the rehabilitation device and power assisting devices has been required based on aging society. However, a flexible actuator that can generate both larger force and longer displacement has not been developed. It is also difficult to realize a flexible displacement sensor with long stroke while deforming its form according to the actuator's shape. In the previous study, the flexible actuator with larger force and longer stroke that can adjust its stroke by giving stepping motion using pneumatic balloons and brakes was proposed and tested. However, the slipping of the pneumatic brake prevents the larger generated force. In this paper, the improved pneumatic brake using a pneumatic balloon and a mechanical chuck is described. The fundamental performance of the actuator using the improved brake is also described.
\end{abstract}

\section{Introduction}

Under the situation of aging society and decreasing birth rate in Japan [1], the development of soft actuator for the rehabilitation device and power assisting devices [2-5] has been required. A rubber artificial muscle is well-known as a soft actuator. Because it generates larger force even if its mass is small. However, the muscle cannot work with longer stroke. The maximum displacement of the muscle is less than one fourth of its original length [6]. In addition, the generated force of the muscle decreases according to the increase of the displacement of the muscle. As a long stroke flexible actuator, a flexible pneumatic cylinder was proposed and tested in the previous study [7]. The cylinder can be used even if it bends. Stroke of the cylinder is able to be set longer according to user's requirement. As applications of the developed cylinder, a flexible robot arm for wrist rehabilitation using three flexible pneumatic cylinders was proposed and tested [8]. A portable rehabilitation device using a spherical actuator that consist of two flexible cylinders was also developed [9]. From these applications, the validity of flexible pneumatic cylinder for application as a rehabilitation device was confirmed $[10,11]$. However, the generated force of the cylinder is not so large. It depends on sectional area of the cylinder. Therefore, it is strongly required to develop a flexible actuator that can generate both larger force and longer stroke. In addition, it is difficult to develop a flexible displacement sensor with long stroke while deforming its form along to the shape of flexible actuator. In ideal, the flexible actuator with larger force and longer stroke that can adjust its stroke without

\footnotetext{
${ }^{a}$ Corresponding author : akagi@are.ous.ac.jp
} 
sensor is desired. To satisfy such a greedy demand, in the previous study, the flexible linear stepping actuator was proposed and tested [12]. It consists of two pneumatic balloons and brakes. The performance of the tested actuator was also investigated. However, the generated force of the tested actuator is not so large. Because holding force of the pneumatic brake is small. In this paper, the improvement of the brake will be described. The performance of whole actuator is also described.

\section{Flexible pneumatic cylinder and previous linear stepping actuator}

In our previous study, the novel flexible pneumatic cylinder as shown in Fig.1 was developed [7]. The cylinder consists of a steel ball with the outer diameter of $9 \mathrm{~mm}$ as a cylinder head, a flexible tube (SMC Co. Ltd., TSU1208) as a cylinder, and a slide stage. The slide stage has two brass rollers set on the inner bore of the stage to press and deform the tube. The roller has an outer diameter of $4 \mathrm{~mm}$. The steel ball is held by two slide stages from both sides of the ball. The operating principle is as follows. When the supply pressure is applied to one side of the cylinder, the tube is moved forward or backward. The cylinder can move with a longer stroke of more than $10 \mathrm{~m}$. It can also move even if the cylinder bends. However, compared with the theoretical generated force of $25 \mathrm{~N}$ at supply pressure of $500 \mathrm{kPa}$, the frictional force of the cylinder is relatively large. It is about $10 \mathrm{~N}$. Therefore, the cylinder can generate the force of about $15 \mathrm{~N}$ under the condition that the supply pressure is $500 \mathrm{kPa}$. Generally, the generated force of the cylinder depends on the sectional area of the cylinder. However, even if the cylinder tube with a larger sectional area is used, the frictional force increases according to increasing the diameter of the cylinder. This is because the contacting area of the sliding mechanism between the inner ball and rollers is increased. In addition, it is difficult to realize a displacement sensor that can measure a longer stroke while changing its shape according to deformation of the cylinder.

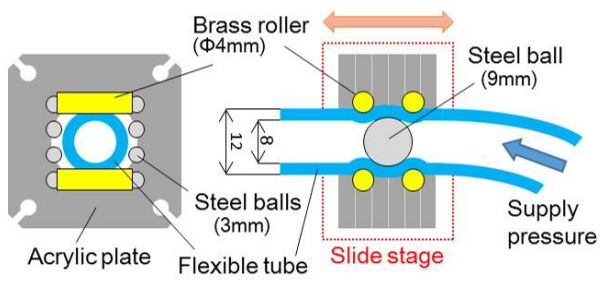

Figure 1. Construction of flexible pneumatic cylinder.
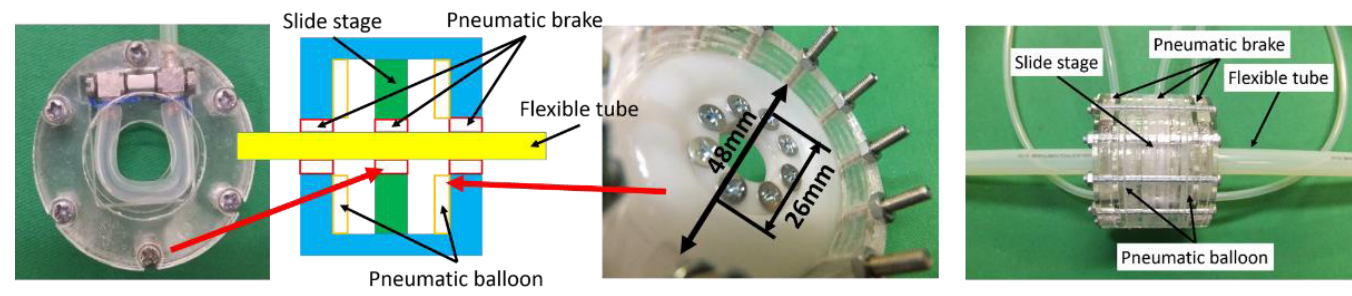

Figure 2. Construction and view of tested flexible linear stepping actuator.

Therefore, in the previous study, a novel pneumatic actuator that can generate larger force and work with a longer stroke was proposed [8]. It can also adjust the displacement without sensor by giving the stepping motion. We called it "a flexible linear stepping actuator". Figure 2 shows the construction and view of the tested actuator. The actuator consists of two doughnut-shaped balloons such as a diaphragm, two pneumatic driven brakes set on both sides of actuator, a moving round stage with a pneumatic driven brake and a flexible tube as a rod. The pneumatic brake consists of silicone rubber tube with outer diameter of $4 \mathrm{~mm}$ surrounded by an acrylic cover. The operating principle of the brake is as follows. When the supply pressure is applied to the silicone rubber tube in the brake, the tube in 
the brake expands. By using the acrylic cover, the rubber tube expands toward the center of the flexible tube and it holds the flexible tube. The moving stage with a brake located on the center of the actuator is sandwiched by two balloons. The doughnut-shaped balloon that consists of a silicone rubber film with thickness of $0.5 \mathrm{~mm}$ has the outer diameter of $48 \mathrm{~mm}$ and the inner diameter of 26 $\mathrm{mm}$. The theoretical generated force calculated from the sectional area of the balloon is about $639 \mathrm{~N}$ when the supply pressure of $500 \mathrm{kPa}$ is applied. The size of the actuator is not so large. It has the length of $50 \mathrm{~mm}$ and the outer diameter of $60 \mathrm{~mm}$. The mass of the actuator without flexible tube (rod) is about $180 \mathrm{~g}$.

The operating principle of the tested actuator is shown in Fig.3. The operating principle is as follows: First, as shown in Fig.3 (1), the both side brakes are driven for $0.2 \mathrm{~s}$ in order to hold the tube. In the condition, the left side balloon is driven for $0.15 \mathrm{~s}$ and the moving stage moves toward right as shown in Fig. 3 (2). When the moving stage reaches at the right side wall by giving the input pressure for 0.15 $\mathrm{s}$, the brake on the stage is activated to hold the tube as shown in Fig.3 (3). After that, while the left side balloon and both side brakes being released, the right side balloon is driven for $0.15 \mathrm{~s}$. So the brake on the moving stage keeps holding the tube, the flexible tube is pushed toward left as shown in Fig. 3 (4). By repeating this procedure, the tube can move toward left every a certain stroke. The stroke is as same as the width of the balloon chamber; that is $5 \mathrm{~mm}$.

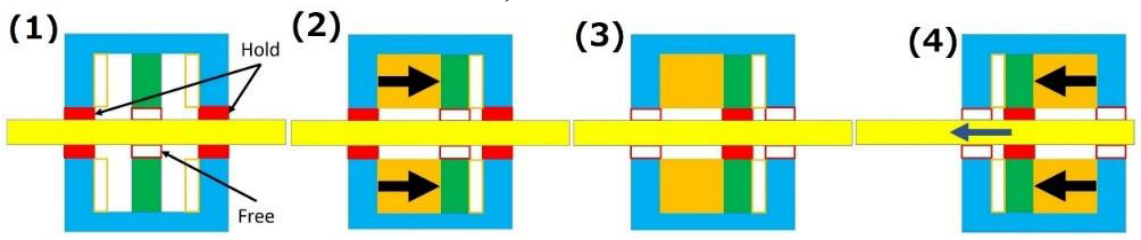

Figure 3. Operating principle of tested flexible linear stepping actuator.

\section{Control system and experimental result of previous actuator}

Figure 4 on the left shows the schematic diagram of the control system of the tested actuator using an embedded controller. Figure 4 on the right shows the time chart of the driving valves. The system consists of the tested actuator, the embedded controller (Renesas Co. Ltd., SH7125) and four on/off control valves (Koganei Co. Ltd., G010E1). In the system, valves 1 and 2 are used to drive both side balloons, respectively. Valve 3 can drive the brake on the moving stage. Valve 4 is used to drive both side brakes. These valves are driven and controlled by the embedded controller through transistors.
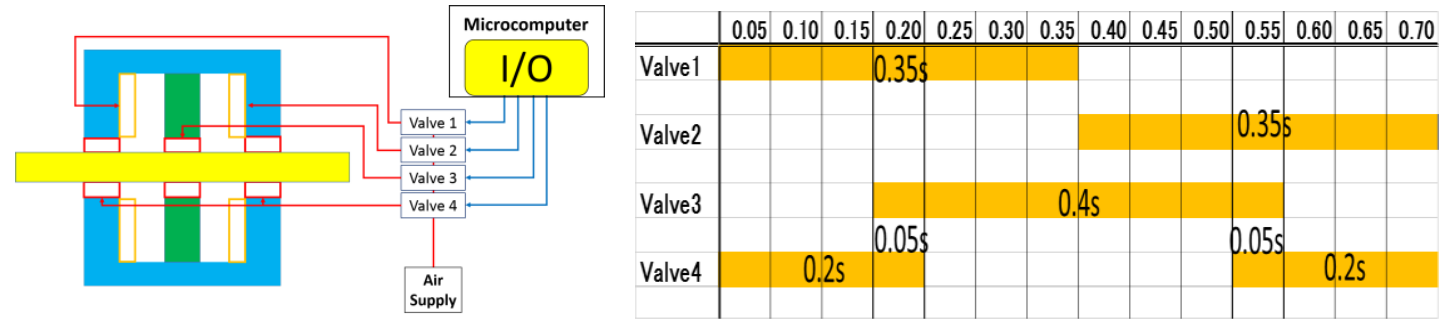

Figure 4. Schematic diagram of control system of tested linear stepping actuator and time chart of driving valves.

Figure 5 shows the transient view of the movement of the tested actuator when the moving pattern of the brakes and balloons are given as shown in Fig. 4 on the right. From the time chart of driving valves, it can be expected that the actuator can push the tube with a step stroke of $5 \mathrm{~mm}$ every $0.7 \mathrm{~s}$. This means the moving speed of the actuator is $7.14 \mathrm{~mm} / \mathrm{s}$. From the experiment as shown in Fig.5, the average speed of the tested actuator was about $5.4 \mathrm{~mm} / \mathrm{s}$. In the experiment, the generated force of the actuator with this moving pattern was also investigated. As a result, the maximum generated force is about $28 \mathrm{~N}$. This value is much smaller than the theoretical value using the area of the balloon. It is caused that the total generated force of the actuator depends on the holding force of the pneumatic 
brake and the generated force of the balloon. In this actuator, as the generated force of the balloon is too enough, the net generated force is equal to holding force of the brake. The theoretical holding force of about $71 \mathrm{~N}$ for radial direction of the brake is calculated from the contacting area of the silicone rubber tube in the brake and the supply pressure of $500 \mathrm{kPa}$. Therefore, with consideration of slip and frictional condition between brake and tube, the generated force of the actuator is less than 71 $\mathrm{N}$. However, this holding force of the brake is too small. Therefore, it is necessary to improve the holding force of the brake.

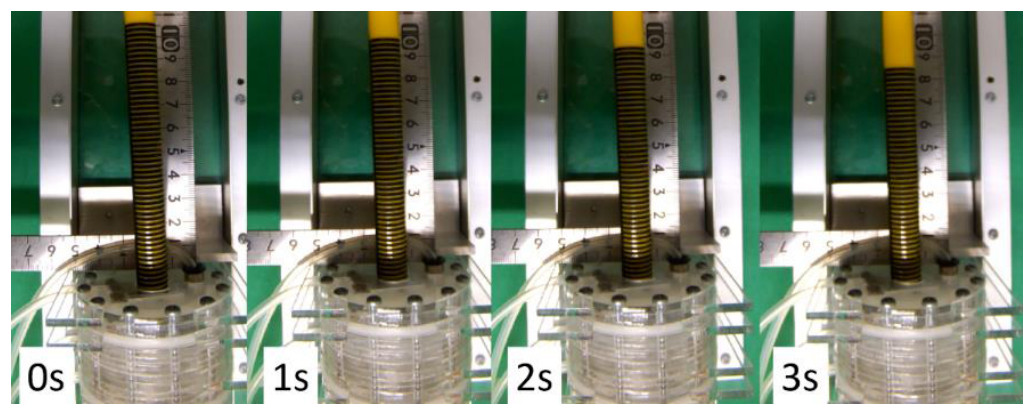

Figure 5. Transient view of movement of the tested actuator.

\section{Improved pneumatic brake and linear stepping actuator}

Figure 6 shows the schematic diagram of the improved pneumatic brake in order to increase the holding force. The improved pneumatic brake consists of a doughnut-shaped balloon with the inner diameter of $24 \mathrm{~mm}$ and the outer diameter of $36 \mathrm{~mm}$, a ring-shaped plastic plate and a mechanical chuck. The mechanical chuck is made of 8 plastic claws set on the stage with the inclined angle of 45 degrees. In the tested chuck, two types of claws were used. One is the claw with width of $6 \mathrm{~mm}$. Another is the claw with width of $3 \mathrm{~mm}$. These two types of claws were used, because the generated braking force was larger than the case using the same size of claws from the preliminary experimental result. In addition, number of the claws was also investigated such as 4,8 and 16 claws. As a result of these preliminary experimental results, it can be found that the alternative arrangement of two types of claw around the flexible tube is suitable to increase the braking force. The inclined angle of $45 \mathrm{deg}$. was calculated as an optimal value theoretically. In the case when the generated force $F$ of the balloon for the brake is applied to the mechanical chuck, the holing force $F_{b}$ obtained by the surrounding pushing force is given as following equation.

$$
F_{b}=\frac{1}{2} F \sin 2 \alpha
$$

Where, $\alpha$ means the inclined angle of the braking stage. From Eq.1, the maximum holding force is given in the case that the inclined angle is $45 \mathrm{deg}$. Then, from the sectional area and supplied pressure of $500 \mathrm{kPa}$, the calculated generated force of the balloon in the brake is about $283 \mathrm{~N}$. Therefore, the maximum generated force of the brake is estimated as less than $141 \mathrm{~N}$. The operating principle of the brake is as follows. When the supply pressure is applied to the balloon, the balloon expands and pushes the ring-shaped plate. The plate pushes eight claws. The claws slide on the inclined stage and hold the flexible tube. By using the balloon with relatively larger sectional area, the holding force will be increased compared with the previous brake.

Figure 7 shows the schematic diagram of the improved linear stepping actuator using the improved pneumatic brake. The fundamental construction of the actuator is almost same as the previous one. Compared with the previous actuator, the actuator has two improved brakes. One is installed between two balloons as a slide stage. Another is set on the outside of the sliding mechanism that consists of two balloons and the slide stage. The size of the actuator without flexible tube becomes larger than 
previous one. The whole length of the actuator is $102 \mathrm{~mm}$ and the outer diameter is same, that is 60 $\mathrm{mm}$. The total mass is about $360 \mathrm{~g}$.

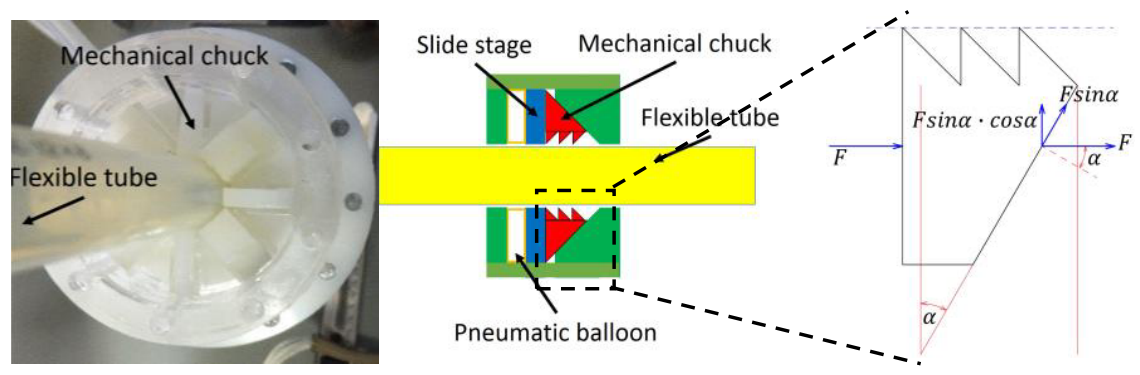

Figure 6. Schematic diagram of improved pneumatic brake using balloon and mechanical chuck.

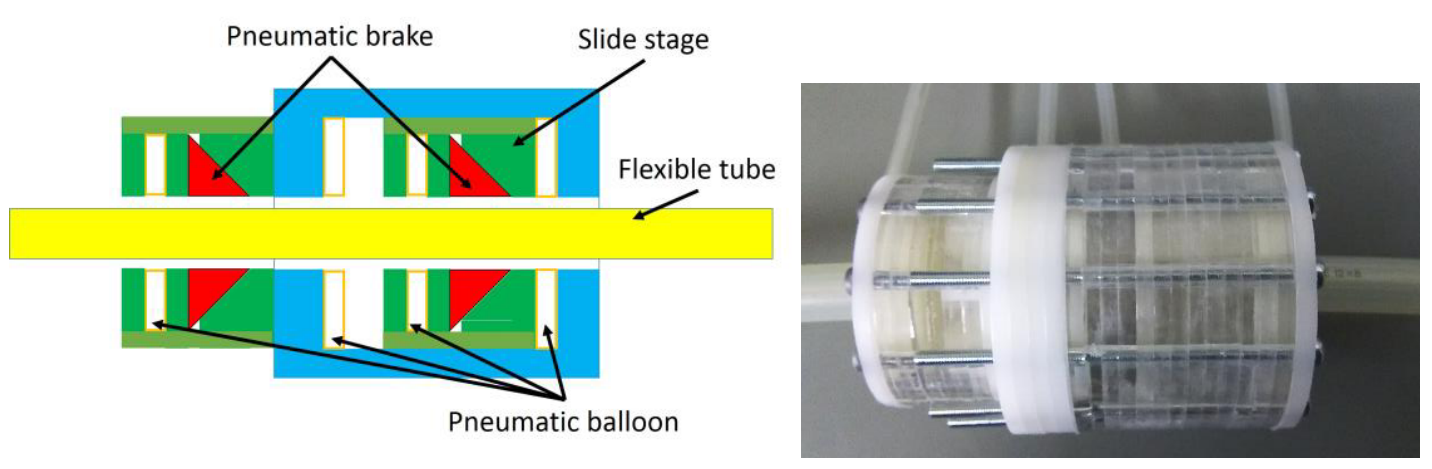

Figure 7. Schematic diagram and view of improved linear stepping actuator.

Figure 8 shows the transient view of movement of the tested actuator when it is lifting up the load of $30 \mathrm{~N}$. In the experiment, the same driving pattern of valves as shown in Fig. 4 was used. From Fig. 8 , it can be seen that the actuator was lifting up the load of $30 \mathrm{~N}$ with average speed of $5 \mathrm{~mm} / \mathrm{s}$. In addition, from the result of the static generated force, it can be found that the maximum generated force of $90 \mathrm{~N}$ can be obtained. This force is about three times larger than that of previous actuator.

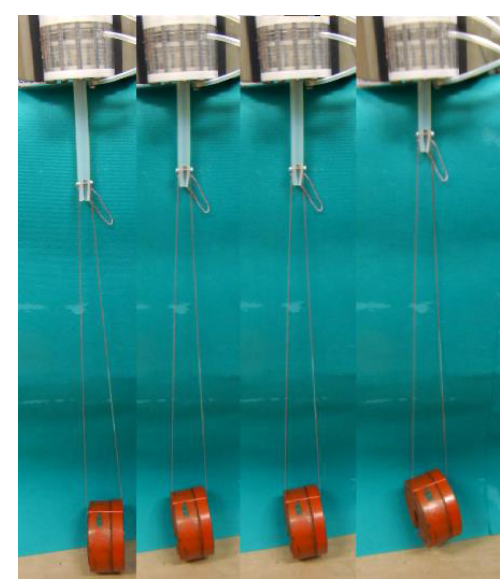

Figure 8. Transient view of the improved actuator when it is lifting the load of $30 \mathrm{~N}$.

\section{Conclusions}


As a flexible actuator with larger force and longer stroke, the previous developed flexible linear stepping actuator driven by pneumatic balloons and brakes was introduced. In order to increase the generated force of the actuator, the improved pneumatic brake that consists of the balloon and mechanical chuck. The linear stepping actuator using the improved brake was also constructed. The moving test lifting up the constant load was carried out. As a result, it can be found that the tested actuator can lift the load of $30 \mathrm{~N}$ with speed of $5 \mathrm{~mm} / \mathrm{s}$ in the experiment. It can be confirmed that the actuator is useful to apply as a rehabilitation device because of larger generated force and constant step stroke of $5 \mathrm{~mm}$.

\section{Acknowledgements}

This research was supported by the Ministry of Education, Culture, Sports, Science and Technology of Japan through a Financial Assistance for QOL Innovative Research (2012-2016) and JSPS KAKENHI Grant Number 16K060202.

\section{References}

1. Ministry of Internal Affairs and Communications, Statistics Bureau, Statistics, Population Estimates, Result of the Population Estimates, Monthly Report. Result of the Population Estimates (online), Available: http://www.stat.go.jp/english/data/jinsui/tsuki/ (2015)

2. M. Ishii, K. Yamamoto, K. Hyodo, Stand-Alone Wearable Power Assist Suit -Development and Availability-. Journal of Robotics and Mechatronics, Vol.17, No.5, 575-583 (2005)

3. J. Piquion, A. Nayar, A. Ghazaryan, R. Papann, W. Klimek, and R. Laroia, Robot-assisted gynecological surgery in a community setting. Journal of Robotics and Surgery, Vol.3, Issue 2, 61-64 (2009)

4. T. Noritsugu, M. Takaiwa, D. Sasaki, Development of Power Assist Wear Using Pneumatic Rubber Artificial Muscles, Journal of Robotics and Mechatronics, Vol. 21, No. 5, 607-613(2009)

5. H. Kobayashi, T. Shiban, Y. Ishida, Realization of all 7 motions for the upper limb by a muscle suit, Journal of Robotics and Mechatronics, Vol. 16, 504-512(2004)

6. Y.Nagata ed., Soft Actuators -Forefront of Development-, NTS Ltd.(2004) (in Japanese)

7. T. Akagi, S. Dohta, Development of a Rodless Type Flexible Pneumatic Cylinder and Its Application, Transactions on Robotics and Automation of the JSME (C), Vol. 73, No. 731, 21082114(2007) (in Japanese)

8. Mohd Aliff, S. Dohta, T.Akagi, H. Li, Development of a simple-structured pneumatic robot arm and its control using low-cost embedded controller, Journal of Procedia Engineering, Vol.41 134 $-142(2012)$

9. S. Dohta, T. Akagi, C. Liu and A. Ando, Development and Control of Flexible Spherical Actuator Using Flexible Pneumatic Cylinders, International Journal of Advanced Mechatronic Systems, Vol. 5, No.3,184- 192(2013)

10. Mohd Aliff, S. Dohta, T. Akagi, T. Morimoto, Control of Flexible Pneumatic Robot Arm Using Master Device with Pneumatic Brake Mechanism, JFPS International Journal of Fluid Power System, Vol.8, No.1, 38-43(2015)

11. Y. Matsui, T. Akagi and S. Dohta, Development and Control of Flexible Spherical Actuator for Portable Rehabilitation Device, International Journal of Materials Science and Engineering, Vol.3, No.1, 49-54(2015)

12. Y. Eguchi, T. Akagi, S, Dohta, Preliminary Design of Flexible Linear Stepping Actuator Driven by Pneumatic Balloons and Brakes, MATEC Web of Conferences, Vol.51,No.02004, 1-4(2016) 